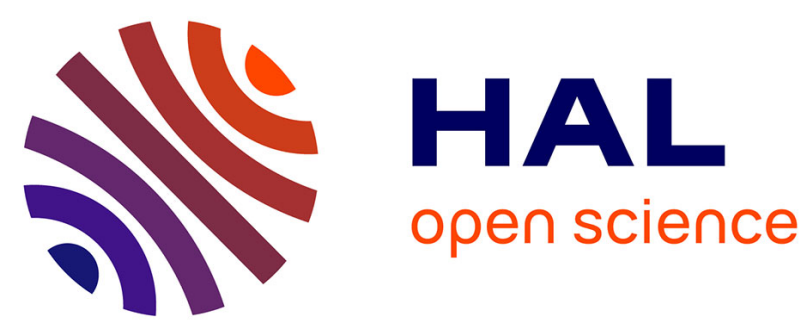

\title{
Experimental and theoretical investigation of the nonmodal growth of steady streaks in a flat plate boundary layer
}

\author{
J.H.M. Fransson, L. Brandt, A. Talamelli, Carlo Cossu
}

\section{To cite this version:}

J.H.M. Fransson, L. Brandt, A. Talamelli, Carlo Cossu. Experimental and theoretical investigation of the nonmodal growth of steady streaks in a flat plate boundary layer. Physics of Fluids, 2004, 16 (10), pp.3627-3638. 10.1063/1.1773493 . hal-01024954

HAL Id: hal-01024954

https://hal-polytechnique.archives-ouvertes.fr/hal-01024954

Submitted on 29 Jul 2014

HAL is a multi-disciplinary open access archive for the deposit and dissemination of scientific research documents, whether they are published or not. The documents may come from teaching and research institutions in France or abroad, or from public or private research centers.
L'archive ouverte pluridisciplinaire HAL, est destinée au dépôt et à la diffusion de documents scientifiques de niveau recherche, publiés ou non, émanant des établissements d'enseignement et de recherche français ou étrangers, des laboratoires publics ou privés. 


\section{AIP $\mid$ Physics of Fluids}

\section{Experimental and theoretical investigation of the nonmodal growth of steady streaks in a flat plate boundary layer}

Jens H. M. Fransson, Luca Brandt, Alessandro Talamelli, and Carlo Cossu

Citation: Physics of Fluids (1994-present) 16, 3627 (2004); doi: 10.1063/1.1773493

View online: http://dx.doi.org/10.1063/1.1773493

View Table of Contents: http://scitation.aip.org/content/aip/journal/pof2/16/10?ver=pdfcov

Published by the AIP Publishing

\section{Articles you may be interested in}

On the relationship between the wall-shear-stress and transient-growth disturbances in a laminar boundary layer Phys. Fluids 22, 054103 (2010); 10.1063/1.3415220

The bifurcation structure of viscous steady axisymmetric vortex breakdown with open lateral boundaries

Phys. Fluids 21, 074107 (2009); 10.1063/1.3176476

Wall heat transfer effects on Klebanoff modes and Tollmien-Schlichting waves in a compressible boundary layer Phys. Fluids 21, 024106 (2009); 10.1063/1.3054155

Development of low-frequency streaks in Blasius boundary layer

Phys. Fluids 16, 3153 (2004); 10.1063/1.1764826

Linear and nonlinear development of localized disturbances in zero and adverse pressure gradient boundarylayers

Phys. Fluids 10, 1405 (1998); 10.1063/1.869665

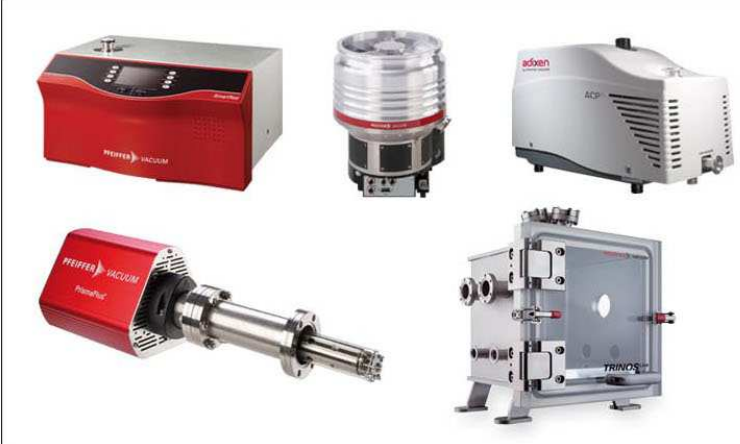

Vacuum Solutions from a Single Source

- Turbopumps

- Backing pumps

- Leak detectors

- Measurement and analysis equipment

- Chambers and components 


\title{
Experimental and theoretical investigation of the nonmodal growth of steady streaks in a flat plate boundary layer
}

\author{
Jens H. M. Fransson and Luca Brandt \\ KTH Mechanics, SE-100 44 Stockholm, Sweden \\ Alessandro Talamelli \\ KTH Mechanics, SE-100 44 Stockholm, Sweden \\ and II Facoltà di Ingegneria, Università di Bologna, 47100 Forlì, Italy \\ Carlo Cossu \\ Laboratoire d'Hydrodynamique (LadHyX), CNRS-Ecole Polytechnique, F-91128 Palaiseau, France
}

(Received 19 January 2004; accepted 27 May 2004; published online 27 August 2004)

\begin{abstract}
An experimental and theoretical investigation aimed at describing the nonmodal growth of steady and spanwise periodic streamwise streaks in a flat plate boundary layer is presented. Stable laminar streaks are experimentally generated by means of a spanwise periodic array of small cylindrical roughness elements fixed on the plate. The streamwise evolution of the streaks is measured and it is proved that, except in a small region near the roughness elements, they obey the boundary layer scalings. The maximum achievable amplitude is mainly determined by the relative height of the roughness elements. Results are compared with numerical simulations of optimal and suboptimal boundary layer streaks. The theory is able to elucidate some of the discrepancies recently noticed between experimentally realizable nonmodal growth and optimal perturbation theory. The key factor is found to be the wall normal location and the extension of the laminar standing streamwise vortices inducing the streaks. The differences among previous experimental works can be explained by different dominating streak generation mechanisms which can be linked to the geometry and to the ratio between the roughness height and the boundary layer scale. (C) 2004 American Institute of Physics. [DOI: 10.1063/1.1773493]
\end{abstract}

\section{INTRODUCTION}

The two-dimensional (2D) Blasius boundary layer developing on a semi-infinite flat plate is known to be linearly unstable to exponentially growing waves in a range of Reynolds numbers and the least stable mode is called the Tollmien-Schlichting (TS) wave. ${ }^{1}$ TS waves modify the basic flow and, when they exceed a critical amplitude of the order of $1 \%$ of the free stream velocity $U_{\infty}$, they experience a secondary instability ${ }^{2}$ which finally leads to fast transition to turbulence. This transition scenario is well understood, at least in its initial part, and is often called the classical transition scenario. This scenario is, however, only observed with low levels of external perturbations. It has been known for many years that the presence of free-stream turbulence or wall imperfections may lead to strong 3D modulations of the boundary layer and trigger early transition. ${ }^{3-5}$

\section{A. Lift-up and streaky structures in 2D boundary layers}

Small amounts of streamwise vorticity are, indeed, very effective in pushing low momentum fluid away from the wall and high momentum fluid towards the wall. This eventually leads to large elongated spanwise modulations of the streamwise velocity field called streamwise streaks. This "lift-up effect" ${ }^{\prime}$ is based on an inviscid mechanism but the effect of viscosity eventually dominates rendering the growth of the streaks only transient. Before their final viscous decay, how- ever, the growth of the streaks may attain values of the order of the Reynolds number. ${ }^{7}$ The potential of shear flows to exhibit such large transient growths is related to the nonnormal nature of the linear stability operator. ${ }^{8}$ The optimal perturbation, i.e., the perturbation leading to maximum transient growth, has been computed for a number of wall bounded shear flows and found to consist of streamwise vortices, while the most amplified perturbation, optimally forced by these vortices, consists of streamwise streaks (for a review the reader may refer to the book by Schmid and Henningson"). The appellation "transient growth" descends from the temporal formulation of the problem where it applies to the growth of low-streamwise-wave-number modes. In a spatial approach, a "similar" behavior is experienced by low-frequency perturbations, consisting of an algebraic growth followed by a slow viscous exponential decay in the streamwise direction. In the nonparallel Blasius boundary layer the disturbance present at the leading edge that is most spatially amplified also consist of spanwise periodic streamwise vortices. The most amplified perturbation consists of streamwise streaks with a spanwise scale of the order of the boundary layer thickness. ${ }^{10,11}$

An extensively studied case of streamwise streaks is the boundary layer developing in the presence of free stream turbulence. In early observations, Dryden ${ }^{12}$ and Taylor ${ }^{13}$ reported that spanwise modulations of the boundary layer thickness are generated in the presence of free stream turbulence. These observations were confirmed and further de- 
tailed by, among others, Arnal and Juillen ${ }^{14}$ and Kendall ${ }^{5}$ who coined the term "Klebanoff mode" for the observed disturbances, referring to early observations by Klebanoff. ${ }^{15}$ Streaks forced by free stream turbulence typically slowly oscillate in the boundary layer in a random way; their wallnormal root mean square (rms) velocity profiles, however, were found to closely match the shape of the steady streak perturbation forced by optimal vortices. ${ }^{10}$ The occurrence of bypass transition observed in the presence of free stream turbulence was therefore related to the stability properties of boundary layers modulated by steady optimal streaks.

\section{B. Influence of streaks on boundary layer stability}

The stability properties of the streaky 3D boundary layers may strongly differ from those of the Blasius 2D boundary layer and depend on the streak amplitude and shape.

For streaks of sufficiently large amplitude the inflection points, appearing in the 3D basic flow, are able to support high frequency secondary instabilities of inviscid nature. Andersson et al. ${ }^{16}$ analyzed the linear inviscid stability of a family of streaky boundary layers parametrized by the amplitude of the linearly optimal vortices introduced at the leading edge of the flat plate. They found that the inflectional instability sets in when the streak amplitude exceeds a critical value of $26 \%$ of $U_{\infty}$.

The viscous stability of the same family of basic flows considered by Ref. 16 has recently been explored in the case of moderate amplitudes of the streaks $\left(<26 \%\right.$ of $\left.U_{\infty}\right)$, which are therefore stable to inviscid instability. It has been found ${ }^{17-19}$ that the streaks have a stabilizing effect on the TS waves, as also observed experimentally by Boiko et $a .^{20}$ in the case of a boundary layer subject to free stream turbulence and unsteady streaks. It was therefore suggested to artificially force such moderate amplitude steady streaks in the Blasius boundary layer in order to delay the onset of the viscous instability, and consequently the transition to turbulence to larger Reynolds numbers.

\section{Experimental generation of steady streaks}

The generation of steady vortices in the flat plate boundary layer has retained the attention of experimentalists since the early 1960s. Most of these investigations aimed at analyzing the effect of streamwise streaks on the boundary layer stability.

In the experiments of Tani and Komoda ${ }^{21}$ and Komoda, ${ }^{22}$ small wings, located outside the boundary layer, were used to generate steady streamwise vortices which, upon entering the boundary layer, led to the development of steady spanwise periodic modulations of its thickness, i.e., to steady streamwise streaks. The circulation of the forced vortices was tuned by changing the incidence of the wings. Streamwise vortices may also be introduced by perturbations applied at the wall of the flat plate, for instance by spanwise periodic blowing and suction through wall slots (see, e.g., Kachanov and Tararykin ${ }^{23}$ among many others). Spanwise periodic arrays of roughness elements have also been proven efficient to introduce steady streamwise vorticity in boundary layers. Roughness elements would probably be simpler to manufacture in case of industrial applications based on the generation of streaks.

Bakchinov et $a l .{ }^{24}$ used a spanwise periodic array of roughness elements of rectangular section, placed at the wall at some finite distance from the leading edge, to generate large amplitude streaks. For low free stream velocities $\left(U_{\infty}\right)$, the amplitude of the streaks was moderate and the streaks were stable up to $20 \%$ of $U_{\infty}$. Far downstream of the roughness array these streaks were periodic, almost sinusoidal in the spanwise direction, and with a wall normal perturbation profile reminiscent of the Klebanoff mode and of the optimal streak shape. Its maximum appeared at $\eta \sim 2 \delta$, where $\delta$ $=\sqrt{\nu x / U_{\infty}}$ is the local boundary layer reference scale at the streamwise station $x$. The high speed streaks were located in spanwise correspondence with the roughness elements, while the low speed streaks were observed to be located in correspondence to the free space between the roughness elements.

White $^{25}$ investigated small amplitude (stable) steady streaks generated by using a spanwise periodic array of roughness elements of circular section and small height. The maximum amplitude of the streaks was below $4 \%$ of $U_{\infty}$. The far downstream shape of the streaks considered by White is also periodic in the spanwise direction and "nearly optimal" in the wall normal direction with, again, a maximum at $2 \delta$. However, in that experiment, the high speed streaks are found to correspond to the space between the roughness elements while the low speed streaks are situated in correspondence to the roughness elements. In Ref. 25 the streamwise evolution of the streaks were carefully analyzed, starting near the roughness array, and the results compared with the characteristics of the numerical streaks arising when optimal vortices are forced at the leading edge. White ${ }^{25}$ found that the experimental streaks differ from the optimal ones in, at least, two important features: (a) the streamwise position at which the peak amplitude is attained is found to be further upstream of the value predicted by optimal theory; (b) the wall normal position of the maximum perturbation velocity of the streak is closer to the wall compared to the prediction by nonmodal growth theory.

\section{Motivation of the present study}

The initial motivation of the present study was to experimentally verify the stabilizing action of streamwise streaks on TS waves that was found by Cossu and Brandt ${ }^{17}$ for optimal streaks of moderate amplitudes $\left(10-25 \%\right.$ of $\left.U_{\infty}\right)$. The results by Ref. 25 showed, however, that the streaks generated by roughness elements are likely to differ from optimal streaks in some important features. The present investigation is therefore aimed at studying, in detail, the evolution of streaks of amplitudes considerably larger than what was analyzed in Ref. 25 and answer the following two relevant questions.

(1) Do the suboptimal trends reported in Ref. 25 for low amplitude streaks apply also to larger amplitude streaks?

(2) What are the main factors affecting the suboptimality of the experimental streaks? 


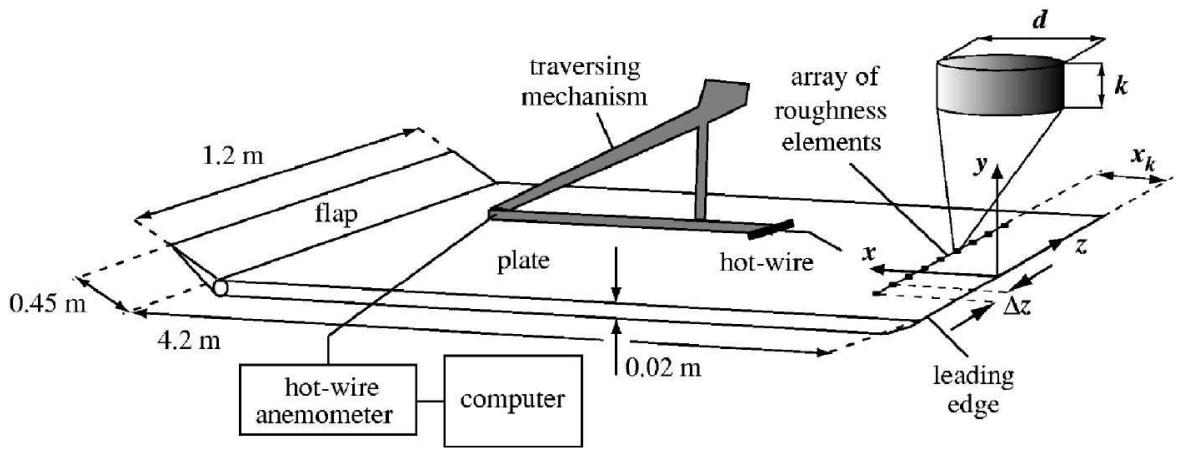

FIG. 1. Schematic of the experimental setup. $k=780 \mu \mathrm{m}, \quad d=2 \mathrm{~mm}, \Delta z$ $=8 \mathrm{~mm}$, and $x_{k}=40 \mathrm{~mm}$.

The answer to the first question is important because the generation process of the large amplitude streaks reported in Ref. 24 seems to differ from that of small amplitude streaks reported in Ref. 25.

It is necessary to answer the second question if one wants to compare the stability of experimental and numerical streaks. Understanding the reasons leading to suboptimality could both allow optimal streaks to be experimentally generated, and the experimental suboptimal streaks to be numerically reproduced. For instance, it is not even a priori known if the experimentally generated streaks verify the boundary layer scalings away from the roughness elements and in that case it would be inappropriate to compare them with optimal streaks which are solutions of the boundary layer equations.

An experimental study was conducted and the results were compared with numerical simulations of both optimal and suboptimal boundary layer streaks in order to answer the above questions. The article is organized as follows: In Sec. II the experimental setting and the particular parameters chosen to generate the streaks are described. In Sec. III the evolution of the experimentally generated streaks is presented and it is proven that they verify the boundary layer scalings except near the roughness elements. It is further checked whether nonlinear saturation effects are important at the considered amplitudes. In Sec. IV the main factors leading to suboptimal behavior are numerically investigated and compared to the experimental findings. In Sec. V the results are discussed and the main findings are finally summarized in Sec. VI.

\section{EXPERIMENTAL SETUP AND PARAMETERS}

\section{A. Test facility}

The experiments were carried out at KTH Mechanics in the Minimum-Turbulence-Level (MTL) wind tunnel, which has a test section of $7 \mathrm{~m}$ in length, $0.8 \mathrm{~m}$ in height, and $1.2 \mathrm{~m}$ in width. The maximum speed is $70 \mathrm{~m} / \mathrm{s}$. After ten years in operation the flow quality of the MTL tunnel has been reconfirmed and the results are collected in an internal technical report by Lindgren and Johansson. ${ }^{26}$ At $25 \mathrm{~m} / \mathrm{s}$ the streamwise turbulence intensity is less than $0.025 \%$ of $U_{\infty}$ and both the cross flow turbulence intensities are less than $0.035 \%$. The tunnel is equipped with a heat exchanger in the return circuit just after the fan which is able to maintain a variation of the total pressure and the temperature below $\pm 0.06 \%$ and $\pm 0.05^{\circ}$, respectively. Furthermore, for boundary layer measurements the tunnel is equipped with a computer controlled five degree of freedom traversing mechanism.

Figure 1 shows a schematic of the experimental setup, with a $4.2 \mathrm{~m}$ long flat plate mounted horizontally in the test section. The plate is the same as described in Klingmann et $a l^{27}$ and has an asymmetric leading edge, in order get a short pressure gradient region without any suction peak at leading edge region. Furthermore, a trailing edge flap is also used in order to regulate the stagnation streamline. The roof of the test section is adjustable making it possible to change the pressure gradient. A zero pressure gradient with a $C_{p}$ variation of less than \pm 0.01 was achieved for this setup. A Cartesian coordinate system is introduced (cf. Fig. 1). The origin is located on the centerline at the leading edge with $x, y$, and $z$ axis directed along the streamwise, wall normal, and spanwise direction, respectively.

\section{B. Experimental setting}

The streaky boundary layer was generated by means of roughness elements made of brass, obtained by cutting slices of a cylinder rod. Several preliminary tests were carried out in order to find the proper shape and dimension for the elements to generate stable and high amplitude streaks. In order to simplify these experiments the roughness elements were positioned in the middle of the plate where the boundary layer is substantially thicker. To get a good spanwise homogeneity nine elements were periodically pasted on the plate surface. Relevant parameters in this study are: the boundary layer scale $\delta$ corresponding to $\sqrt{x \nu / U_{\infty}}$, the height of the roughness element $k$, the cylinder diameter $d$, the spacing between the elements $\Delta z$, and the distance from the leading edge where the elements were located $x_{k}$. In the present setting the fixed parameters are $k=780 \mu \mathrm{m}, d=2 \mathrm{~mm}, \Delta z$ $=8 \mathrm{~mm}$, and $x_{k}=40 \mathrm{~mm}$ (see Fig. 1 for an illustration of all parameters).

A single hot-wire probe operating at constant temperature was used to measure the streamwise velocity component (here denoted $u$ ). The probe was built at KTH Mechanics and was made of a $2.5 \mu \mathrm{m}$ platinum wire with a distance between the prongs of approximately $0.5 \mathrm{~mm}$. The calibration function proposed by Johansson and Alfredsson ${ }^{28}$ was used, where an extra term is added to King's law for the compensation of natural convection making it suitable for low speed measurements. No temperature compensation is required due 
to the presence of the heat exchanger and temperature control system. The probe was operated at $50 \%$ overheat and was calibrated against a Prandtl tube in the free stream.

\section{Choice of parameters}

Wind tunnel experiments were performed at different free stream velocities, viz. $5,6,7$, and $8 \mathrm{~m} / \mathrm{s}$ while keeping the same height, streamwise, and spanwise locations, and diameter of the roughness elements. It should be mentioned that throughout this paper $\delta$ has been calculated as $\sqrt{x \nu / U_{\infty}}$. Comparison with the integrated measured velocity profiles, in the absence of roughness elements, resulted in a virtual origin of the streamwise coordinate of approximately $-5.8 \mathrm{~mm}$, independent of the free stream velocity, which has here been judged as small and has therefore not been accounted for in the presented data. Changing the free stream velocity is one way of regulating the streak amplitude since it influences the height to the boundary layer scale ratio $(k / \delta)$. A stronger spanwise modulation of the boundary layer flow is achieved with an increase of velocity and vice versa. When $k / \delta$ or alternatively the roughness element Reynolds number $\operatorname{Re}_{k}=u(y=k) k / \nu$ becomes too large transition to turbulence occur. The critical value of $\mathrm{Re}_{k}$ for transition depends on the roughness element geometry and the flow quality of the wind tunnel, i.e., the background disturbance level and reported values are found in the range 300-1000 (cf. Klebanoff $e t a l .^{29}$ ).

The selected spanwise distance between the roughness elements $(\Delta z)$ sets the generated spanwise scales. However, a large $\Delta z$ will induce an extra streak in between the roughness elements and initially there will be a mismatch between $\Delta z$ and the spanwise wavelength of the energy dominating mode. The dimensionless fundamental spanwise wave number is often defined as $\beta=2 \pi \delta / \Delta z$. However, $\Delta z$ does not necessarily correspond to the dominating mode, which makes the definition delicate since comparisons are often made with the $\beta$ predicted by optimal perturbation theory in where the spanwise modulation of the base flow only consists of one mode. For the present choice of parameters the dominating/fundamental spanwise wavelength $\left(\lambda_{0}\right)$, as will be shown, will correspond to $\Delta z$ for $x \geqslant 70 \mathrm{~mm}$.

The Reynolds number based on the local Blasius scale ( $\delta)$ at $x_{k}\left(\operatorname{Re}_{\delta}=U_{\infty} \delta / \nu\right)$ is a relevant stability parameter, and the critical value for amplifying TS waves is well known to be around 300. Numerous test measurements with the goal to generate stable, high amplitude, and sinusoidal streaks, were made before the particular parameters given in the caption of Fig. 1 were chosen. The parameters effected by the change of free stream velocity are summarized in Table I.

\section{EXPERIMENTAL RESULTS}

\section{A. Perturbations induced by the roughness elements 1. Evolution in physical space}

To follow the initial stage of the streak development, results for the configuration with $U_{\infty}=7 \mathrm{~m} / \mathrm{s}$ are first presented. The stationary streamwise velocity perturbation is shown in the cross-stream $(y, z)$ plane at different streamwise
TABLE I. Summary of the experimental parameters. $A^{*}$ is the maximum streak amplitude in percentage of $U_{\infty}$. $\delta$ is evaluated at the position of the roughness elements $x=x_{k}$.

\begin{tabular}{ccccccc}
\hline \hline$U_{\infty}(\mathrm{m} / \mathrm{s})$ & $\delta(\mathrm{mm})$ & $k / \delta$ & $R e_{k}$ & $R e_{\delta}$ & $\beta$ & $A^{*}(\%)$ \\
\hline 5 & 0.35 & 2.24 & 180 & 115 & 0.272 & 5.5 \\
6 & 0.32 & 2.46 & 231 & 126 & 0.248 & 7.5 \\
7 & 0.29 & 2.65 & 285 & 137 & 0.230 & 9.5 \\
8 & 0.27 & 2.84 & 340 & 146 & 0.215 & 11.5 \\
\hline \hline
\end{tabular}

stations in Fig. 2. The perturbation velocity is defined as the difference of the local velocity $U(y, z)$ to the spanwiseaveraged profile $U(y)$. Contours of constant streamwise velocity are also shown for comparison. Note that a region of large velocity excess is formed further downstream $(x$ $\geqslant 70 \mathrm{~mm}$ ) straight behind the cylinders, located at $z=0$ and $8 \mathrm{~mm}$. The decay phase of the streaks is shown in the contour plot $500 \mathrm{~mm}$ downstream of the leading edge. The spanwise profiles change into almost sinusoidal curves as the disturbance evolves downstream and the details of the flow induced just behind the roughness elements are lost. As observed also in Ref. 25, a complicated disturbance is found in the region closest to the roughness array, in which the wake of each element is transformed into the periodic spanwise

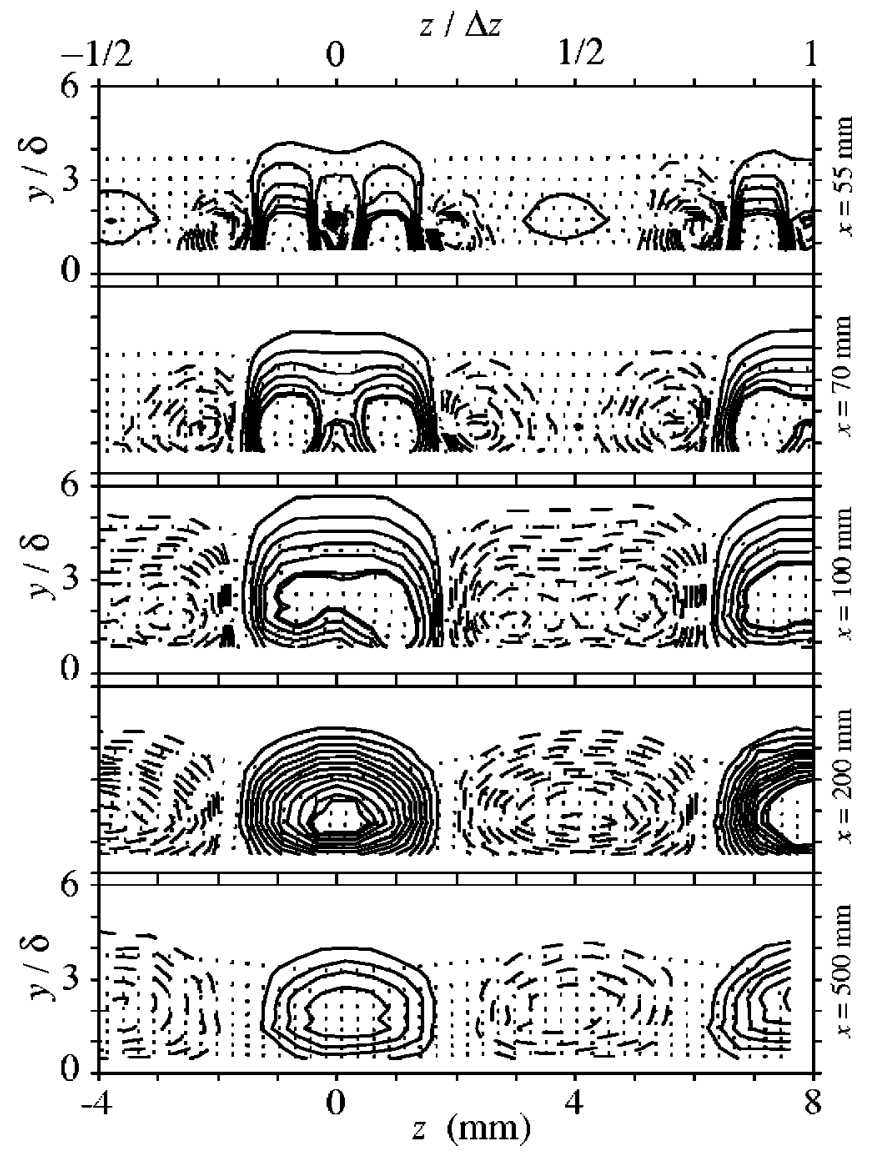

FIG. 2. Streamwise evolution of positive and negative streamwise velocity perturbations (solid and dashed lines, respectively). Dotted contour lines $\left(0.1 U_{\infty}\right.$ of increment) correspond to constant streamwise velocity. $U_{\infty}$ $=7 \mathrm{~m} / \mathrm{s}$. 


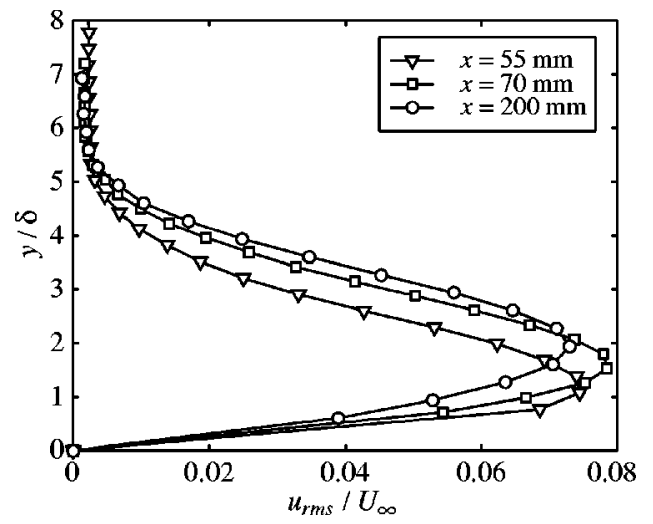

FIG. 3. Disturbance profiles in the wall-normal direction for different downstream locations. $U_{\infty}=7 \mathrm{~m} / \mathrm{s}$.

modulation of the streamwise velocity observed farther downstream. In the present experiments as well as in Ref. 25 standing cylinder roughness elements have been used, however, in the former case a high speed streak is formed straight behind the element (in agreement with Refs. 24 and 30) and in the latter case a low speed streak is achieved (in agreement with Refs. 31 and 32). The explanation of the contra- dictory observations is that there are different dominating generation mechanisms present (cf. Sec. V A for an extension of the subject). However, both mechanisms give rise to an algebraic growth followed by a viscous decay of the energy with the downstream distance.

In Fig. $3 u_{\text {rms }}$ profiles, based on the spatial $U(z)$ distribution, for three different downstream locations are compared. The positions are $x=55,70$, and $200 \mathrm{~mm}$ corresponding to 51,103 , and 548 boundary layer scales $\delta$ downstream of the elements. It is clear from the figure that the roughness elements induce a single peak in the $u_{\text {rms }}$ profile, which is in accordance with other experiments. ${ }^{24,30,34}$

\section{Evolution of the spanwise harmonics}

Normalized spanwise velocity profiles at the wallnormal position of maximum perturbation are displayed in Fig. 4. Here, the evolution into an almost sinusoidal modulation of the base flow in the downstream direction is illustrated, and the higher harmonics induced straight behind the roughness elements become negligible. The power spectral density (PSD) of the considered profiles are also shown in Fig. 4. The dominating wavelength is dictated by the roughness array periodicity already at $x>70 \mathrm{~mm}$ or beyond 103
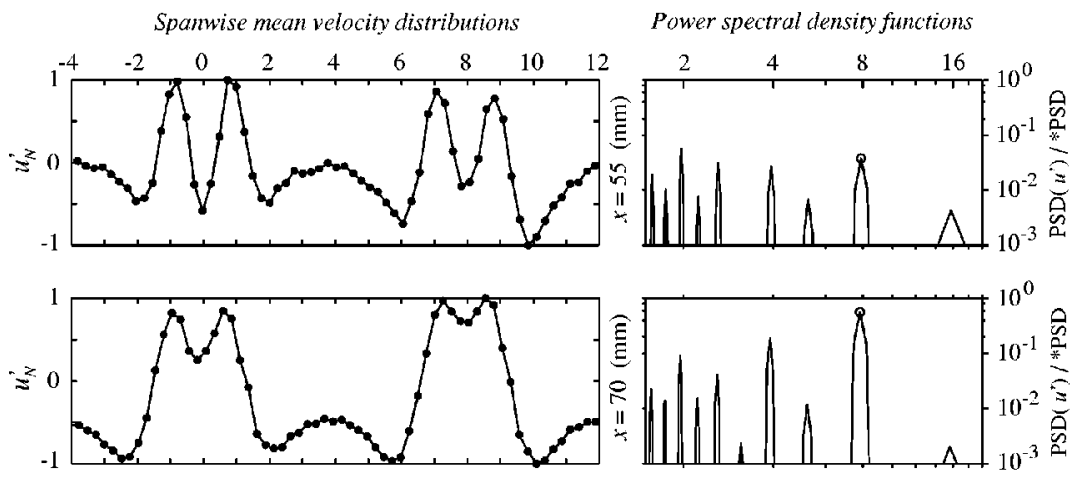
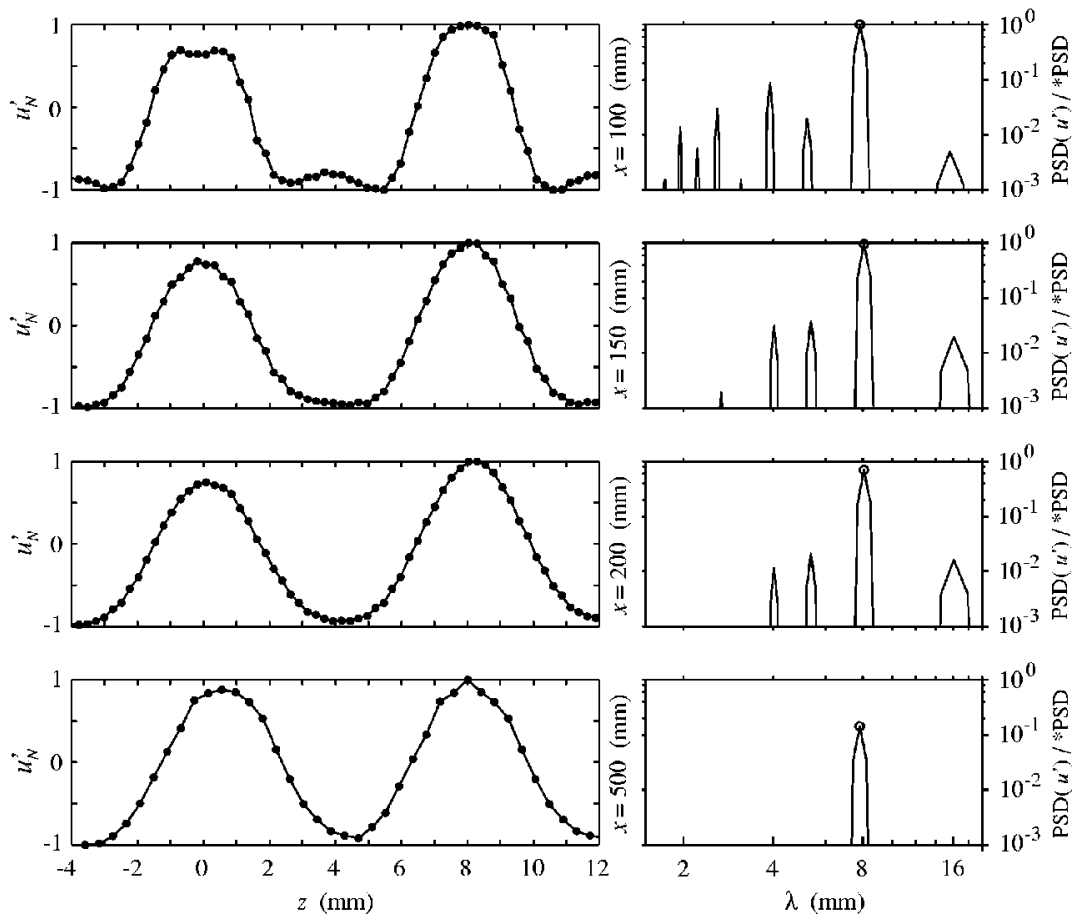

FIG. 4. Streamwise evolution of the mean spanwise velocity profile taken at the wall-normal position corresponding to the disturbance peak. (a) Spanwise velocity distribution normalized to \pm 1 , and (b) spanwise power spectral density function. The normalization is such that ${ }^{*} \mathrm{PSD}$ corresponds to $\max \left\{\operatorname{PSD}\left(u^{\prime}\right)\right\}$ for all $x$. $U_{\infty}$ $=7 \mathrm{~m} / \mathrm{s}$. 

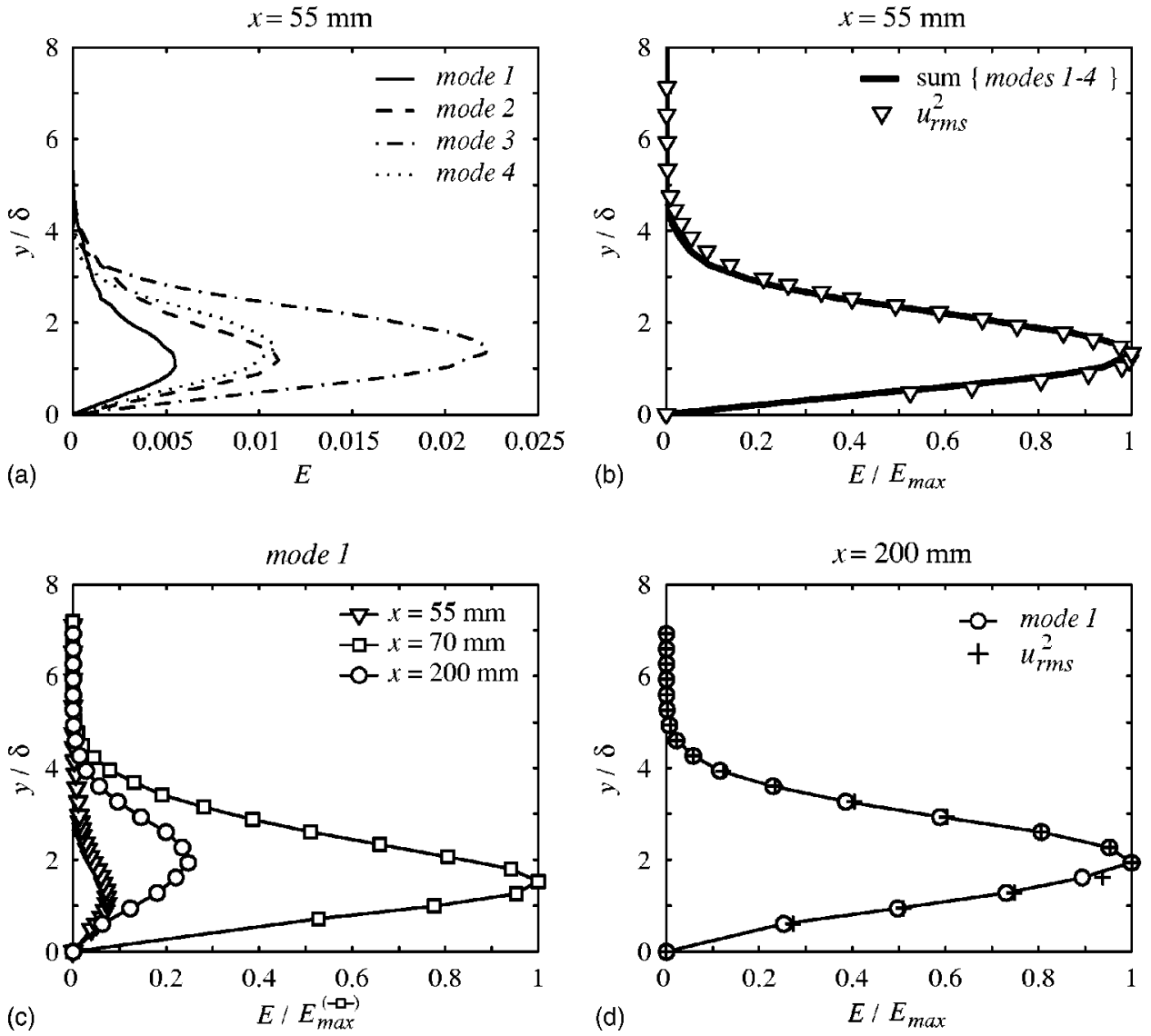

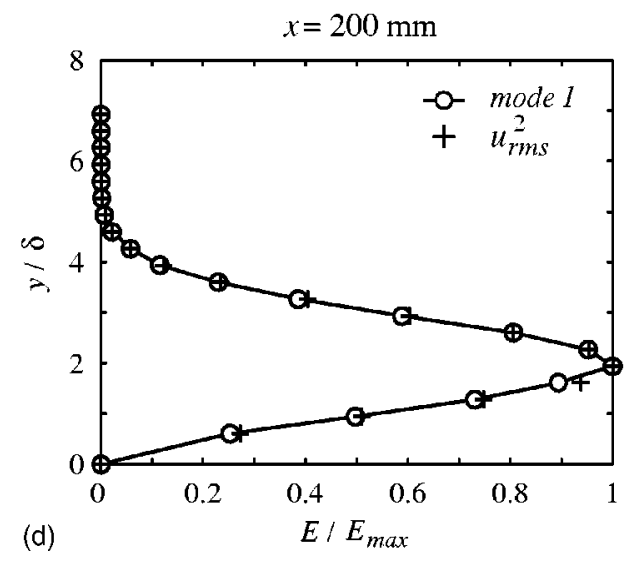

FIG. 5. Decomposed energy mode profiles in the wall-normal direction. $U_{\infty}=7 \mathrm{~m} / \mathrm{s}$. (a) Power spectral density distributions for the fundamental (1) mode $\lambda_{0}=8 \mathrm{~mm}$ and higher harmonics; (2) $\lambda_{0} / 2$, (3) $\lambda_{0} / 4$, and (4) $\lambda_{0} / 5$. (b) Comparison between the $u_{\mathrm{rms}}^{2}$ (Fig. 3) and the superposition of all four modes. (c) Fundamental mode for different $x$ positions. (d) Comparison between the $u_{\text {rms }}^{2}$ (Fig. 3) and the fundamental mode. boundary layer scales downstream of the elements. Relatively small energy peaks at the higher harmonics of the fundamental wavelength $\left(\lambda_{0}=8 \mathrm{~mm}=\Delta z\right)$ can also be seen. These harmonics reach their respectively energy maxima upstream the fundamental mode which is in concordance with theory and the results by Ref. 25. Note here that if the PSD figures in Fig. 4 were to be plotted with a linear scale, as in Ref. 25, the fundamental mode would appear clearly dominating already from $x=70 \mathrm{~mm}$.

In Fig. 5 the PSD distributions in the wall-normal direction are shown for different modes. Figure 5(a) shows the four modes with most energy content, which correspond to the wavelengths $\lambda_{0}=8 \mathrm{~mm}, \lambda_{0} / 2, \lambda_{0} / 4$, and $\lambda_{0} / 5$. The superposition of these four modes in Fig. 5(a) is able to represent the $u_{\text {rms }}$ profile (see Fig. 3) computed in physical space, this is illustrated in Fig. 5(b), where the two profiles are compared. Figure 5(c), displaying the evolution of the fundamental mode in the downstream direction, shows that the peak position moves away from the wall in terms of $y / \delta$. Finally, in Fig. 5(d) the fundamental mode is compared with the $u_{\text {rms }}$ profile at the same $x$ position, namely, $x=200 \mathrm{~mm}$, and shows excellent agreement. This implies that the higher harmonics are negligible and that the nonlinear effects are weak. Generally, higher harmonics can be induced both by nonlinearities and by the fact that the roughness elements do not produce a purely sinusoidal disturbance. From the evolution of the spectra we can deduce that, in the present case, the higher harmonics present are initially due to the generation process while further downstream they are the result of weak nonlinearities.

\section{Streamwise evolution of the streak amplitude}

In this section, the downstream evolution of the streaks for the four cases analyzed, i.e., $U_{\infty}=5,6,7$, and $8 \mathrm{~m} / \mathrm{s}$, is considered. It is shown that, except the region just downstream of the roughness, most of the perturbation energy is in the fundamental harmonic and the streak amplitude can therefore be considered in the physical space. The streak amplitude $A_{\mathrm{ST}}$ is defined as the wall-normal maximum of the peak-to-peak difference between the streamwise velocities in the low and high speed regions, i.e.,

$$
A_{\mathrm{ST}}=\max _{y}\left\{U(y)_{\text {high }}-U(y)_{\text {low }}\right\} / 2,
$$

where $U(y)_{\text {high }}$ and $U(y)_{\text {low }}$ are the velocity profiles in the high and low speed regions, respectively.

The streamwise evolution of the amplitude $A_{\mathrm{ST}}$ is displayed in Fig. 6(a). For the same experimental setup, stronger streaks are induced by increasing $U_{\infty}$. This is both due to the increase of the cylinder height relative to the local boundary layer displacement thickness and to the increased velocity impinging on the elements. Furthermore, it is observed that the location of maximum amplitude moves downstream with increasing $U_{\infty}$. In Fig. 6(b), the downstream evolution of the wall-normal position of the maximum of the steady perturbation is shown for the four cases. The observed waviness of this wall-normal position is probably due to the lack of measurement points around the maximum. Despite the fact that the values have been obtained by fitting a second order polynomial to the points around the maximum, the evolution in the downstream direction appears 

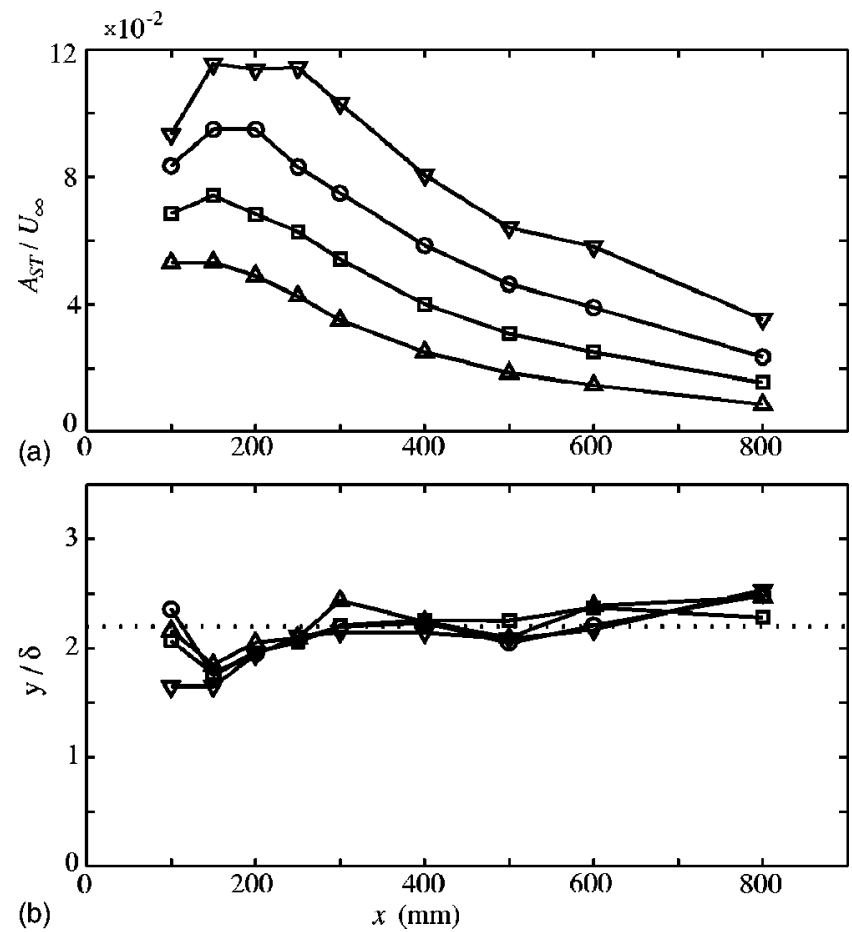

FIG. 6. Streamwise evolution of the streaks: (a) Streak amplitude $A_{\mathrm{ST}}$ vs $x(\mathrm{~mm})$. (b) Wall-normal position of the maximum streak amplitude normalized with the local Blasius length scale $\delta$. The symbols $(\triangle),(\square),(\bigcirc)$, and $(\nabla)$ correspond to $U_{\infty}=5,6,7$, and $8 \mathrm{~m} / \mathrm{s}$, respectively.

slightly wavy, something that also can be observed in Ref. 25 . The dotted line represents the theoretical value obtained for the optimal perturbation at the leading edge of the flat plate $y / \delta=2.2$ (see Refs. 10 and 11). In all four cases the position of maximum perturbation is seen to move upwards in the boundary layer which is in agreement with the experiments by White. ${ }^{25}$

\section{B. Boundary layer scaling and linearity of the streaks}

The data in Fig. 6 can be rescaled according to the usual boundary layer scaling to check whether the experimentally observed streaks also satisfy this scaling. This property is based on the assumption of slow streamwise variation of the flow and it is therefore not valid in the vicinity of the roughness elements. However, the downstream evolution of the perturbations can still be described by the boundary layer equations. As shown in Refs. 10,11, and 16, steady streamwise streaks obey the boundary layer approximation and are therefore Reynolds number independent. This results in a scaling property that couples the spatial scales of the problem to the free stream velocity and viscosity, implying that a similar streak profile is obtained for every combination of $x \nu \beta^{* 2} / U_{\infty}=$ constant, where $\beta^{*}=2 \pi / \Delta z$. A universal streak behavior can be retrieved if we chose a nondimensional $x$ coordinate defined as $X=\mathcal{C} \cdot x \nu \beta^{* 2} / U_{\infty}$, where we select $\mathcal{C}$ such that at $X=1$ the spanwise wave number is the optimal one $\left(\beta_{\text {opt }}=0.45\right)$ predicted by theory. ${ }^{10,11}$ This $x$ scaling can also be interpreted as $X=\left(\beta^{*} \delta / \beta_{\mathrm{opt}}\right)^{2}$, which clearly shows
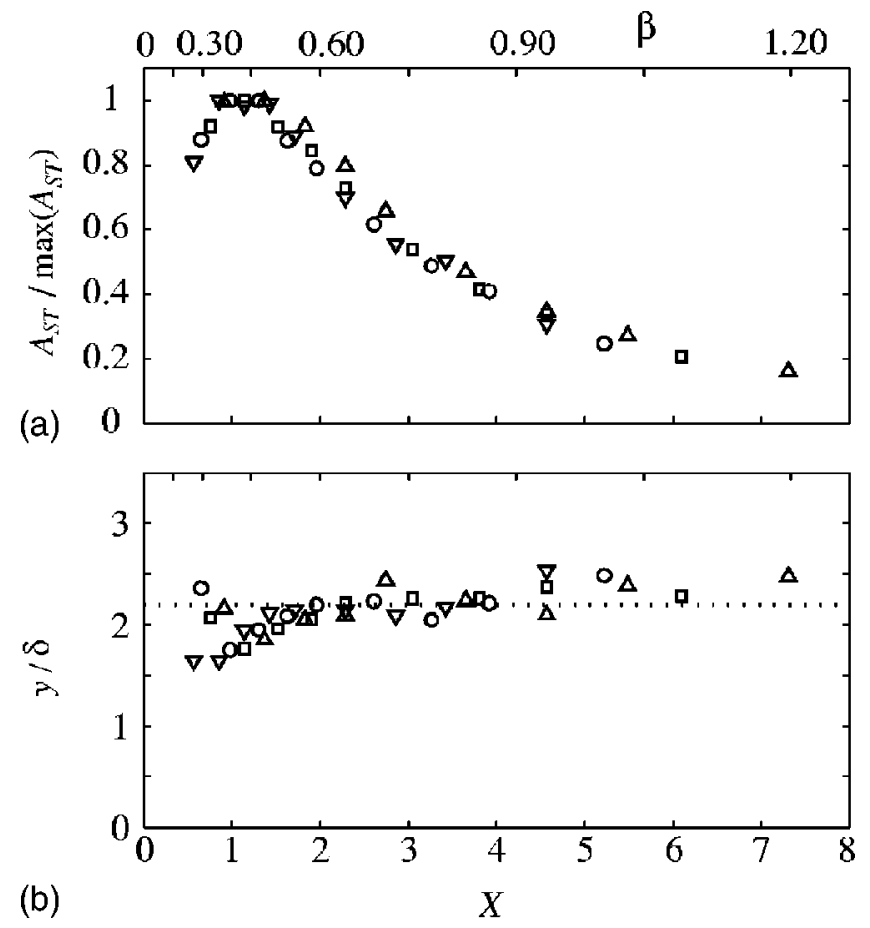

FIG. 7. Streamwise evolution of the streaks: (a) Streak amplitude $A_{\mathrm{ST}}$ normalized to unity vs the boundary layer streamwise coordinate $X$ and the local spanwise wave number $\beta=2 \pi \delta / \Delta z$. (b) Wall-normal position of the maximum streak amplitude normalized with the local Blasius length scale $\delta$. Symbols are same as in Fig. 6.

that the universal streak will only be observed if the relevant flow parameters remain constant when scaled with $\delta$, i.e., if locally all the streaks have the same $\beta$.

The result of applying this streamwise coordinate $X$ to the experimental data is shown in Fig. 7, where the streak amplitude $A_{\mathrm{ST}}$ is normalized with its streamwise maximum. The collapse of the different curves is remarkable despite that the local $\beta$, at the position of the roughness elements, slightly differs for the different realizations. It should also be noted that the four configurations considered in Fig. 6 differ in the value of $k / \delta$, i.e., the relative height of the roughness elements. In principle, this would break the suggested scaling property. However, it does not affect the curve collapse in the plot, where the amplitude of the streak is divided by its maximum value. Therefore, it may be inferred that the parameter $\mathrm{k} / \delta$ mainly determines the maximum achievable streak amplitude. The maximum amplitude is attained for a local $\beta$ of about 0.45 in all cases and the evolution of the wall-normal position of the maximum perturbation is also in agreement among the different experimental configurations [Fig. 7(b)]. From the collapse of the curves it can also be concluded that the streaks behave linearly and that the nonlinear interactions are not strong enough to induce the upstream shift of the location of the maximum amplitude noted in the simulations in Ref. 16 for high-amplitude nonlinearly saturated streaks. Hence, the differences between optimal perturbation and experimentally generated streaks cannot be explained by nonlinearities, as expected by the results in Ref. 25 where the importance of nonlinear terms could be ruled out due to the small amplitudes of the experimental streaks. 


\section{SUBOPTIMALITY OF THE EXPERIMENTAL STREAKS}

The streak amplitude curves in Fig. 6 show only a qualitative agreement with the predictions from nonmodal growth theory: the maxima occur farther upstream, thus at lower local $\beta$ than for the optimal disturbances, as observed also in Ref. 25 , and the decay downstream of the maximum is faster. In order to understand the physical reason for the observed differences, numerical simulations are performed on a simple theoretical model.

\section{A. Numerical method}

The numerical code used in Andersson et al. ${ }^{10}$ was used as a basis for the present simulations. That code was developed to study the evolution of perturbations elongated in the streamwise direction and it is therefore based on the boundary-layer approximation to the steady, linearized, incompressible Navier-Stokes equations. The spanwise dependence of such perturbations is assumed to be periodic and the only spanwise harmonic considered is the fundamental.

The optimal perturbations are obtained by an adjointbased optimization technique. As measure of the perturbation size the kinetic energy at a specific streamwise location is considered. The obtained optimal disturbance maximizes the output disturbance energy at the final location $X_{f}(=1)$ among all possible inputs at $X=X_{0}$ of fixed energy. The upstream optimal disturbance is computed by power iterations, applying the direct and adjoint operators on the initial guess (for a detailed description see Ref. 10).

The numerical scheme adopted to solve the boundary layer and the corresponding adjoint equations uses Chebychev polynomials in the wall-normal direction and a fully implicit finite difference scheme in the marching/streamwise direction. In particular, a second-order backward-difference scheme is implemented, except for the first backward-Euler step.

\section{B. Influence of the streamwise position of the initial perturbation}

Since the perturbation is introduced by the roughness elements a certain distance downstream of the leading edge, the code used in Ref. 10 has been modified to optimize for initial conditions at a generic streamwise station $X_{0}$ different from the leading edge. The interested reader is referred to Levin and Henningson ${ }^{35}$ for a thorough account of the effect of the initial position $X_{0}$ on the transient growth of streaks. In particular, these authors show that the optimal perturbation introduced at a certain position downstream of the leading edge gives rise to a larger growth than the optimal perturbation at the leading edge.

The optimal perturbation for the experimental flow parameters at $U_{\infty}=7 \mathrm{~m} / \mathrm{s}$, corresponding to $X_{0}=0.2611$ and $\beta_{0}=0.230$, has been computed and is considered in this section. Figure 8 shows the comparison between the evolution of the wall-normal maximum of the streamwise velocity perturbation for the optimal perturbation at the leading edge, the optimal perturbation at the actual location of the roughness element, and the measured data. Considering the numerical

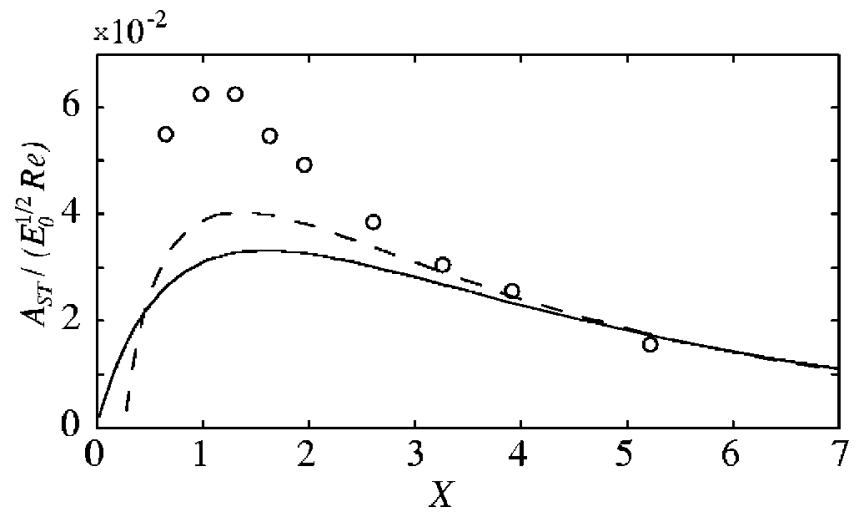

FIG. 8. Streamwise evolution of the wall-normal maximum of the streamwise velocity perturbation of the optimal perturbations introduced at the leading edge (solid line) and at the actual position of the roughness elements $X_{0}=0.2611$ (dashed line). The open circles indicate the measured streak amplitude $A_{\mathrm{ST}}$, scaled to compare the final decay rates.

results, it can be seen that the growth is larger for the case of initial optimal perturbation at $X_{0}=0.26$, in agreement with the findings in Ref. 35. The discrepancies between experimental and theoretical results are qualitatively similar in the two cases, i.e., in the experiments the position of maximum growth occurs slightly closer to the leading edge and the downstream decay is initially faster. The experimental data are normalized in the plot so as to emphasize that farther downstream $(X>4)$ the decay rate becomes similar in all cases. It can therefore be concluded that the generation position of the initial perturbation cannot alone explain the observed streak behavior.

\section{Influence of the shape of the initial perturbation}

The optimal perturbation at $X_{0}=0.2611$ has zero streamwise velocity and therefore it consists only of streamwise vorticity $\omega_{x}$. The latter is shown by the solid line in Fig. 9. It can be seen in the figure that the maximum of $\omega_{x}$ is located far up in the boundary layer, namely at $y / \delta=3.55$. It was therefore decided to study the effect of changing the position of the initial streamwise vortices relative to the wall and to

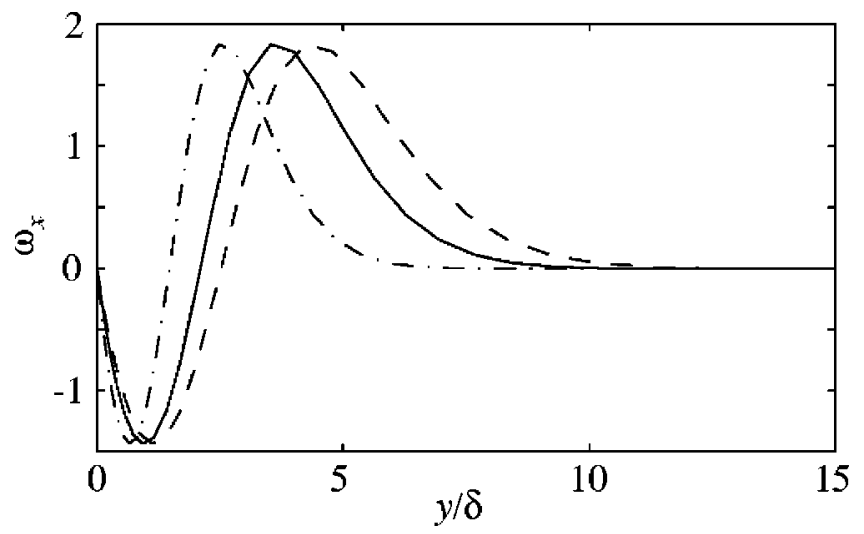

FIG. 9. Wall-normal distribution of the streamwise vorticity of optimal disturbance at $X_{0}=0.2611, \beta_{0}=0.2299$ (solid line). Suboptimal initial conditions obtained by stretching or compressing the optimal disturbance by a factor $c$. Dashed line: $c=1.2$; dash-dotted line: $c=0.7$. 


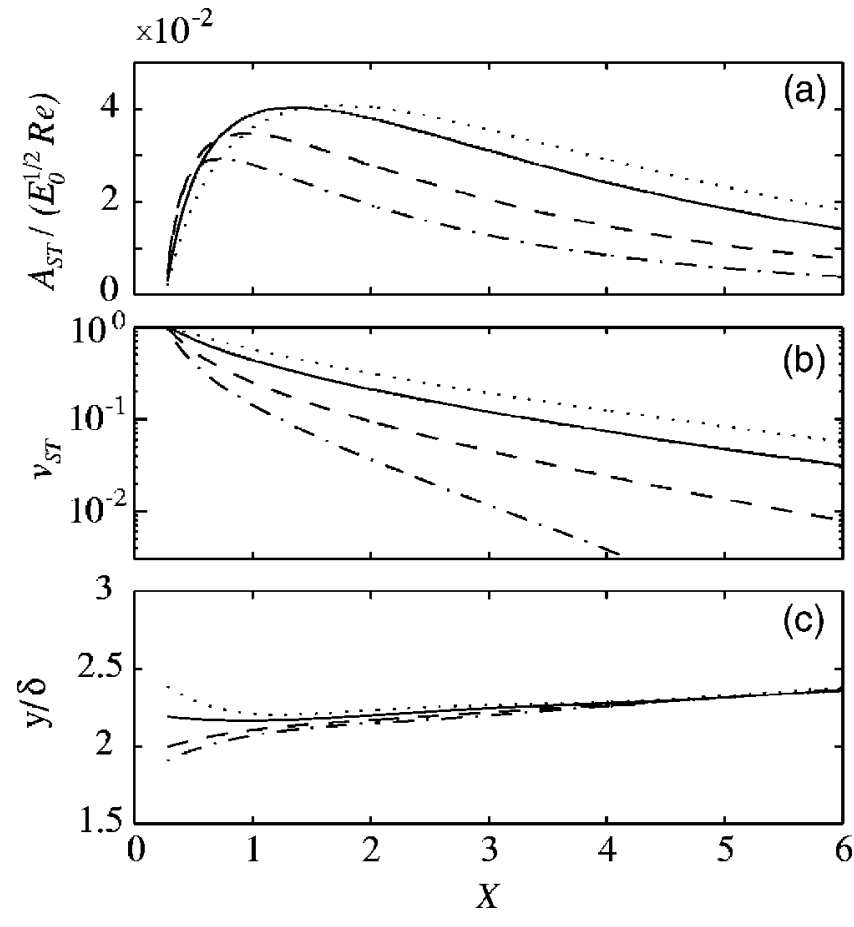

FIG. 10. Streamwise evolution of the streak generated by initial conditions at $X_{0}=0.2611, \beta_{0}=0.2299$. (a) Streamwise velocity amplitude; (b) wallnormal maximum of the wall-normal velocity amplitude; and (c) wallnormal maximum of the streamwise velocity perturbation. Solid line: $c=1$ (optimal disturbance); dotted line: $c=1.2$; dashed line: $c=0.8$; dash-dotted line: $c=0.7$.

verify if this could be the cause of the differences between experimental data and optimal perturbations. Since $u=0,{ }^{10,35}$ $w$ may be derived directly from $v$ through the continuity equation, and the wall-normal profile of $v$ or alternatively of $\omega_{x}$ completely defines the optimal disturbance at the upstream position $X_{0}$. The wall-normal profiles of the suboptimal initial vortices considered below are also shown for comparison in Fig. 9. These different conditions are obtained simply by stretching or compressing the wall-normal velocity profile of the optimal upstream perturbation. This amounts to multiply the wall-normal coordinate $(y)$ pertaining to the optimal perturbation by a factor $c$, so that the position of the maximum is moved to $y / \delta=3.55 c$.

It should be noted that this is the simplest approximation among the many possible initial conditions, which can be used to represent the perturbation induced by a wall-mounted cylinder. In particular, considering the discussion below on the generation mechanism, the wall-normal vorticity associated to the cylinder wake is not considered. Only the streamwise vorticity of the standing vortex forming upstream of the obstacle is taken into account. However, as shown below, this approximation allows some essential features of the streak generation process to be captured.

The downstream evolution of the wall-normal maximum of the streamwise velocity, corresponding to the same streak amplitude definition as in the experiments, is displayed in Fig. 10(a) for different values of the parameter $c$. The curve pertaining to the optimal perturbation (optimal at $X=1$ ) is indicated by the solid line. For values of the parameter $c<1$, i.e., for initial streamwise vortices confined closer to the wall, lower amplification is obtained. Furthermore, the location of maximum amplitude is moving upstream and a faster decay is observed downstream of the maximum. These effects become even clearer for lower values of $c$. Conversely, for $c>1$, i.e., for an initial vortex farther away from the wall, the location of the maximum growth moves downstream. The vortex needs a longer distance to diffuse into the growing boundary layer and to interact with the wall-normal shear of the basic flow. It is also interesting to note that the peak value is slightly larger for $c=1.2$. Considering the energy growth (not shown here), even larger differences are observed among the different cases, all in the same trend shown by considering the streak amplitude. The maximum of the wall-normal velocity $V_{\mathrm{ST}}$ is displayed in Fig. 10(b), where the logarithmic axis is chosen to emphasize the final decay rates. It is observed that the decay is exponential and that the increase in the parameter $c$, corresponding to the increase in the wall-normal scale of the upstream vortices, is associated with a decrease of the downstream decay of the perturbations. This is in agreement with previous theoretical studies on the evolution of free stream perturbations; the modes of the continuous spectrum of the linearized Navier-Stokes equations for a parallel boundary layer flow which show the lowest decay rate are in fact characterized by the largest wall-normal scales. ${ }^{36}$ Note that for low-frequency perturbations, the modes of the continuous spectrum are not zero inside the boundary layer (see Hultgren and Gustavsson ${ }^{37}$ among others). The faster decay rate of the streamwise vortices can therefore explain both the reduced streak growth and the faster final decay.

The wall-normal position of the maximum of the streamwise velocity perturbation in Fig. 10(a) is shown in Fig. 10(c). After an initial phase in which the position of the maximum seems to be related to the location of the upstream vortices, the streak is slightly displaced away from the wall, in agreement with the experimental data. It is also interesting to note in Fig. 10(a) that, proceeding further downstream, the decay rates of the streak amplitudes become much closer to each other. Furthermore, it is also possible to show that for $X>4$ the wall-normal profiles of the perturbation velocity $u$ do not only have their maxima at the same $y / \delta$, as shown in Fig. 10(c), but they also fall on top of each other. This is also true for the optimal initial condition at the leading edge ${ }^{10,11}$ if the boundary layer equation are integrated down to values of $X>4$. Hence, sufficiently downstream of the generation station, the shape of the streamwise velocity perturbation becomes independent of the initial vortices, while the amplitude is still affected by the optimality of the upstream input. The streaks can be considered as a "pseudomode" triggered in the boundary layer (see, e.g., Luchini ${ }^{11}$ ).

In Fig. 11 the best fit to the experimental data is shown. This is obtained for a value of $c=0.78$, corresponding to the location $y / \delta=2.75$ of the maximum of the streamwise vorticity perturbation at the position of the roughness elements. This value is remarkably close to the height of the roughness element $k / \delta=2.65$. In Fig. 11(a) the streak decay is well captured while the position of maximum amplification is slightly upstream compared to the experiments. This discrepancy is probably due to the more complicated perturbation 

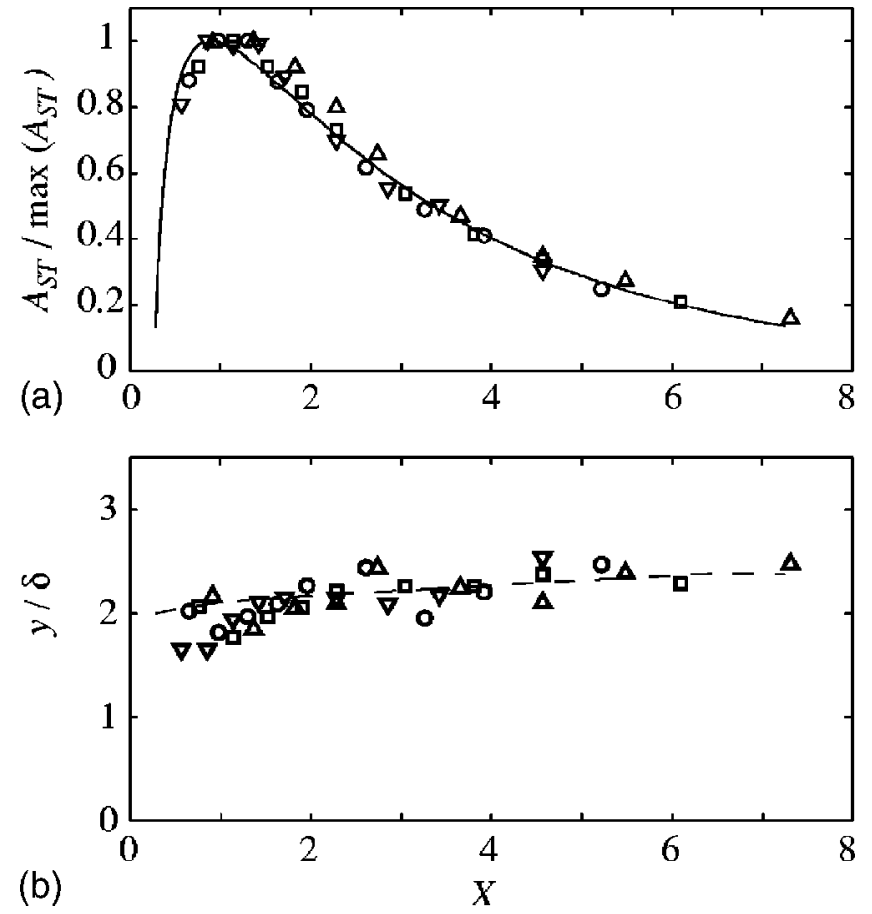

FIG. 11. Comparison between the experimental results for all $U_{\infty}$ in Table I and the best fitting to $U_{\infty}=7 \mathrm{~m} / \mathrm{s}$ obtained using initial vortices confined closer to the wall $(c=0.78)$. (a) Wall-normal maximum of the streak amplitude. (b) Wall-normal position of the maximum perturbation.

pattern observed just downstream of the elements. Recall (Fig. 4) the presence of significant energy in different spanwise harmonics of the perturbation in the wake region close to the cylinders. The downstream increase of the position of maximum perturbation is also well predicted [see Fig. 11(b)].

Finally, the wall-normal distribution of the streamwise velocity perturbation at two different streamwise stations is compared with the computations of suboptimal initial condition using $c=0.78$ in Fig. 12. The experimental streak profile is defined as the peak-to-peak difference in the high and low speed regions. The plot shows a good agreement with the theoretical result.

From the results of this section it can be concluded that the distance to the wall and the extension of the fundamental

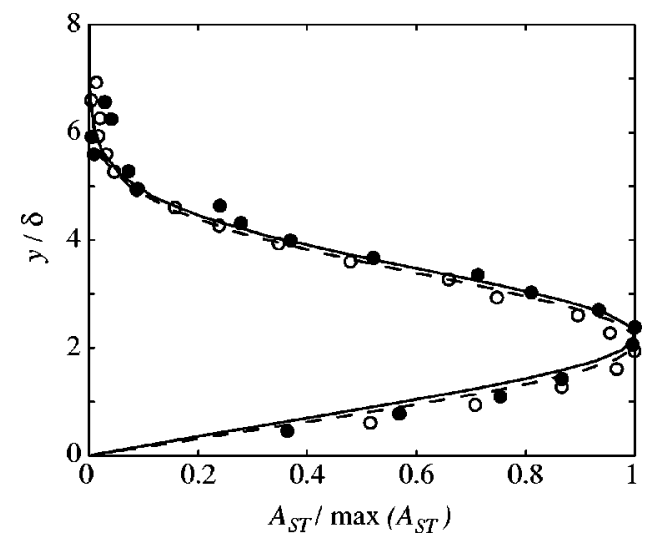

FIG. 12. Wall-normal perturbation profiles at $x=200$ and $500 \mathrm{~mm}$ (or $X$ $=1.31$ and 3.26) corresponding to unfilled and filled symbols, respectively. Dashed and solid lines are the corresponding theoretical profiles. vortices is an essential parameter for the generation of steady streaks. Vortices confined into the boundary layer induce streaks, which grow less, reach their peak amplitude upstream, and decay faster if compared to the optimal perturbation. Conversely, vortices displaced away from the wall create streaks, which reach higher peak amplitude but at positions further downstream. This latter type of initial condition seems more difficult to achieve with wall-mounted actuators. The factor which is able to explain the difference between optimally growing perturbations and experimentally induced streaks has thus been identified, both in the present experiment and in the study of Ref. 25 , in spite of the different configurations.

\section{DISCUSSION}

\section{A. Vorticity generation mechanism on the roughness elements}

Comparison of the above results with similar experimental studies in the literature shows that different flow configurations can be induced by the presence of roughness elements. They are all characterized by the formation of streamwise elongated velocity perturbations and differ in the relative position of the high and low speed streaks with respect to the roughness elements. For instance, in the experiments by Gaster et al. ${ }^{31}$ and related simulations by Joslin and Grosch, ${ }^{32}$ Ref. 25, and Asai et al. ${ }^{38}$ a region of defect velocity is formed straight behind the element. This is due to the presence of the wake, which persists downstream forming the low speed streak. Conversely, in the present experiment, similarly to what was observed by Ref. 24 and Kendall, ${ }^{30}$ after a complex region of growing and decaying modes a high speed region is induced behind the roughness element.

An explanation for this behavior can be found by considering the perturbation induced by a roughness element in a wall-bounded shear flow. ${ }^{39-41}$ The spanwise vorticity of the incoming shear flow is wrapped around the front part of the obstacle forming a steady horseshoe-shaped vortex structure with the two streamwise legs pointing downstream. The vorticity associated with these two counterrotating streamwise vortices is such that high speed fluid is pushed towards the wall in the region behind the obstacle and low speed fluid is lifted on the outer sides. ${ }^{39,41}$ The amount of vorticity concentrated in the streamwise legs of the standing vortex depends on the relative height of the element with respect the boundary layer thickness $(k / \delta)$.

It must be pointed out that the experiments in Ref. 40 show a more complicated flow pattern. Indeed, depending on the top edge geometry of the elements and for sufficiently high values of $\mathrm{Re}_{k}$, a shedding of periodic hairpin vortices may be present. The legs of each vortex consist of two counterrotating streamwise vortices, which in this case increase the defect velocity induced in the wake and generate relatively high levels of velocity fluctuations (larger than 5\% of $\left.U_{\infty}\right)$. Nevertheless, the low level of the streamwise velocity fluctuations (less than $0.6 \%$ of $U_{\infty}$ ) and the absence of any power spectra peak show that this phenomenon is not present in the current experiment. 


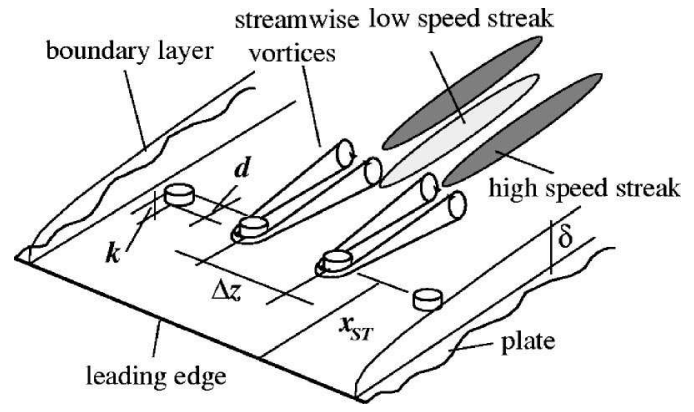

FIG. 13. Schematic of the roughness elements disposition and conceptual view of the streaks generation mechanism for the present case.

It can be concluded that there is a competition between the perturbation induced by the vortex generated by the incoming vorticity upstream of the element and the wake downstream of it. In the present experiment the former mechanism is most likely to dominate and a high speed region is located in correspondence with the roughness element. This can be inferred from Fig. 2 (see also the sketch in Fig. 13): a region of defect velocity (wake) is induced just behind the elements, $z=0$ and $8 \mathrm{~mm}$ and $x=55 \mathrm{~mm}$.

The streamwise legs of the standing vortex cannot be seen from the streamwise velocity contour plot but their effect seems evident further downstream. The two vortices push high-momentum fluid from the upper part of the boundary layer towards the wall in the region behind the roughness elements, so that the region of defect velocity present at $x$ $=55 \mathrm{~mm}$ is first annihilated and then replaced by the high speed streak $(x=70$ and $100 \mathrm{~mm})$. The two vortices lift up low-momentum fluid on their sides; they start to interact with the vortices induced by the neighboring cylinders already at $x=100 \mathrm{~mm}$ so as to induce a low speed region centered at $z=-4$ and $4 \mathrm{~mm}$, see plot at $x=200 \mathrm{~mm}$ in Fig. 2 where well-formed streaks are shown. The same scenario has recently been put forward by Tumin and Reshotko ${ }^{42}$ in the linear approximation. These authors show that the flow behind a three-dimensional hump possesses a pair of streamwise vortices that bring down high speed fluid, whereas the streamwise velocity perturbation in the wake is negative. Downstream of the hump, the velocity perturbation changes its sign due to the down-wash motion induced by the vortices. They also note that after that point, the perturbation experiences the transient growth.

Conversely, in the experiments by Refs. 25, 31, and 32, any given element generates a wake associated with a counterrotating wall-normal vortex pair which decay downstream. In these cases, the streamwise vorticity of the vortex forming upstream of the obstacle is not able to overcome the defect velocity of the wake.

The studies mentioned above are all characterized by different configurations, both in terms of the shape of the roughness elements, the ratio between their spanwise width to their height, and the local Reynolds number. However, a close examination seems to indicate that the ratio $k / \delta$ is the most relevant parameter: the wake mechanism is seen to be the most relevant for low values of $k / \delta$, while the standing vortex is dominating for larger values of $k / \delta$. Note also that, if the latter mechanism is dominating, streaks of higher amplitude are induced.

Finally, it is interesting to note that, in the present case, the spanwise wavelength of the streaks is unambiguously determined by the distance between the roughness elements (see Fig. 4). In Ref. 25 and in White and Ergin, ${ }^{43}$ on the contrary, the spanwise dimension (i.e., the diameter) of the roughness elements determines the dominating spanwise scale of the disturbace downstream. In Ref. 25 the spacing of the roughness elements is $12.5 \mathrm{~mm}$ and the fundamental spanwise wavelength is half of this value, while in Ref. 43 similar cylindrical elements (diameter of $6.35 \mathrm{~mm}$ ) are placed $19 \mathrm{~mm}$ apart. In the latter case the most energetic wavelength is one third of the spacing. For both experimental configurations the dominating scale is about $6 \mathrm{~mm}$, remarkably close to the spanwise width of the elements and to the width of the induced wake.

\section{B. Effect of suboptimality for streaks induced by free-stream turbulence}

The behavior of the suboptimal initial conditions presented in Sec. IV C is also believed to be able to explain the differences observed between optimal perturbation theory and streaks induced by free stream turbulence. It is in fact observed in Matsubara and Alfredsson ${ }^{44}$ that the streaks forced by free stream perturbations are characterized by a spanwise wave number slightly larger than the one predicted by linear optimal perturbation theory. ${ }^{10,11}$ Fransson and Alfredsson $^{45}$ experimentally confirmed these results and showed that for increasing levels of free stream turbulence the experimental results approach the theoretical. The present results show that vortices extending further above the boundary layer than the optimal vortices reach their maximum amplitude further downstream and are therefore associated to larger values of the local wave number $\beta$ at the saturation stage. Assuming these higher vortices a better representation of free stream turbulence, the narrower spanwise scales observed in the experimental studies mentioned above can be explained.

\section{SUMMARY AND CONCLUDING REMARKS}

Steady, spanwise periodic streamwise streaks have been experimentally generated in a flat plate boundary layer using a spanwise array of small roughness elements of cylindrical section. All the streak growth curves are found to collapse when rescaled on the maximum streak amplitude and on the local spanwise wave number. The experimentally generated streaks are hence found to satisfy the similarity property valid for perturbations obeying the boundary layer approximation. Moreover, it has previously been observed, see Ref. 25 , that discrepancies exist between the streaks induced by an array of roughness elements and the optimal streaks, particularly in the streamwise and wall-normal positions of the maximum streak amplitude. The same differences are observed for the large amplitude streaks generated in the present work. By comparing experimental data and numerical simulations, it has been found that these discrepancies 
can be attributed to the streamwise vortices generated by the roughness elements: the latter are more confined into the boundary layer than the theoretically predicted optimal vortices. By considering suboptimal vortices, theoretical predictions and experimental streak amplitude curves are seen to agree. In view of applications in the boundary layer transition delay, as recently suggested in Ref. 17, it has also been shown that it is possible to experimentally generate streaks of moderate amplitude, larger than those considered in Ref. 25 , which are still stable to inflectional secondary instability.

\section{ACKNOWLEDGMENTS}

We wish to thank Professor Henrik Alfredsson and Professor Dan Henningson for helpful discussions and suggestions and Marcus Gällstedt for his assistance in the roughness elements manufacturing. C.C. acknowledges financial support from CNRS during his stay at KTH. The financial support of the Swedish Research Council (V.R.) is also acknowledged.

${ }^{1}$ H. Schlichting, Boundary-Layer Theory (McGraw-Hill, New York, 1979).

${ }^{2}$ T. Herbert, "Secondary instability of boundary-layers," Annu. Rev. Fluid Mech. 20, 487 (1988).

${ }^{3}$ P. S. Klebanoff, K. D. Tidstrom, and L. Sargent, "The three-dimensional nature of boundary layer instability," J. Fluid Mech. 12, 1 (1962).

${ }^{4}$ I. Tani, "Boundary layer transition," Annu. Rev. Fluid Mech. 1, 169 (1969).

${ }^{5}$ J. M. Kendall, "Experimental study of disturbances produced in a pretransitional laminar boundary layer by weak free-stream turbulence," AIAA Pap. 85-1695 (1985).

${ }^{6} \mathrm{M}$. T. Landahl, "A note on an algebraic instability of inviscid parallel shear flows," J. Fluid Mech. 98, 243 (1980).

${ }^{7}$ L. H. Gustavsson, "Energy growth of three-dimensional disturbances in plane Poiseuille flow," J. Fluid Mech. 224, 241 (1991).

${ }^{8}$ L. N. Trefethen, A. E. Trefethen, S. C. Reddy, and T. A. Driscoll, "Hydrodynamic stability without eigenvalues," Science 261, 578 (1993).

${ }^{9}$ P. J. Schmid and D. S. Henningson, Stability and Transition in Shear Flows (Springer, New York, 2001).

${ }^{10}$ P. Andersson, M. Berggren, and D. S. Henningson, "Optimal disturbances and bypass transition in boundary layers," Phys. Fluids 11, 134 (1999).

${ }^{11} \mathrm{P}$. Luchini, "Reynolds-number independent instability of the boundary layer over a flat surface. Part 2: Optimal perturbations," J. Fluid Mech. 404, 289 (2000).

${ }^{12}$ H. L. Dryden, "Air flow in the boundary layer near plate," NACA Report (1937).

${ }^{13}$ G. I. Taylor, "Some recent developments in the study of turbulence," in Proceedings of the Fifth International Congress on Applied Mechanics, edited by J. P. Den Hartog and H. Peters (Wiley, New York, 1939), p. 294.

${ }^{14} \mathrm{D}$. Arnal and J. C. Juillen, "Contribution expérimentale à l'étude de la reptivité d'une couche limite laminaire à la turbulence de l'écoulement général," ONERA, Rapport Technique Nr. 1/5018 (1978).

${ }^{15}$ P. S. Klebanoff, "Effect of free-stream turbulence on the laminar boundary layer," Bull. Am. Phys. Soc. 10, 1323 (1971).

${ }^{16}$ P. Andersson, L. Brandt, A. Bottaro, and D. S. Henningson, "On the breakdown of boundary layer streaks," J. Fluid Mech. 428, 29 (2001).

${ }^{17}$ C. Cossu and L. Brandt, "Stabilization of Tollmien-Schlichting waves by finite amplitude optimal streaks in the Blasius boundary layer," Phys. Fluids 14, L57 (2002).

${ }^{18}$ L. Brandt, C. Cossu, J.-M. Chomaz, P. Huerre, and D. S. Henningson, "On the convectively unstable nature of optimal streaks in boundary layers," J. Fluid Mech. 485, 221 (2003).

${ }^{19}$ C. Cossu and L. Brandt, "On Tollmien-Schlichting-like waves in streaky boundary layers," Eur. J. Mech. B/Fluids (to be published).

${ }^{20}$ A. V. Boiko, K. J. A. Westin, B. G. B. Klingmann, V. V. Kozlov, and P. H. Alfredsson, "Experiments in a boundary layer subjected to free stream turbulence. Part 2. The role of TS-waves in the transition process," J. Fluid Mech. 281, 219 (1994).

${ }^{21}$ I. Tani and H. Komoda, "Boundary layer transition in the presence of streamwise vortices," J. Aerosp. Sci. 29, 440 (1962).

${ }^{22} \mathrm{H}$. Komoda, "Nonlinear development of disturbance in a laminar boundary layer," Phys. Fluids 10, S87 (1967).

${ }^{23}$ Y. S. Kachanov and O. I. Tararykin, "Experimental investigation of a relaxating boundary layer," Izv. SO AN SSSR, Ser. Tech. Nauk 18, 9 (1987).

${ }^{24}$ A. A. Bakchinov, G. R. Grek, B. G. B. Klingmann, and V. V. Kozlov, "Transition experiments in a boundary layer with embedded streamwise vortices," Phys. Fluids 7, 820 (1995).

${ }^{25} \mathrm{E}$. B. White, "Transient growth of stationary disturbances in a flat plate boundary layer," Phys. Fluids 14, 4429 (2002).

${ }^{26} \mathrm{~B}$. Lindgren and A. V. Johansson, "Evaluation of the flow quality in the mtl wind-tunnel," KTH/MEK Report No. TR-02/13-SE, 2002.

${ }^{27}$ B. G. B. Klingmann, A. V. Boiko, K. J. A. Westin, V. V. Kozlov, and P. H. Alfredsson, "Experiments on the stability of Tollmien-Schlichting waves," Eur. J. Mech. B/Fluids 12, 493 (1993).

${ }^{28}$ A. V. Johansson and P. H. Alfredsson, "On the structure of turbulent channel flow," J. Fluid Mech. 122, 295 (1982).

${ }^{29}$ P. S. Klebanoff, W. G. Cleveland, and K. D. Tidstrom, "On the evolution of a turbulent boundary layer induced by a three-dimensional roughness element," J. Fluid Mech. 237, 101 (1992).

${ }^{30}$ J. M. Kendall, "The effect of small-scale roughness on the mean flow profile of a laminar boundary layer," in Instability and Transition, edited by M. Y. Hussaini and R. G. Voigt (Springer, New York, 1990), p. 296.

${ }^{31}$ M. Gaster, C. E. Grosch, and T. L. Jackson, "The velocity field created by a shallow bump in a boundary layer," Phys. Fluids 6, 3079 (1994).

${ }^{32}$ R. D. Joslin and C. E. Grosch, "Growth characteristics downstream of a shallow bump: Computation and experiment," Phys. Fluids 7, 3042 (1995).

${ }^{33}$ E. B. White and F. G. Ergin, "Using laminar-flow velocity profiles to locate the wall behind roughness elements," Exp. Fluids 36, 805 (2004).

${ }^{34}$ Note that, as explained by White and Ergin (Ref. 33) the two peak $u_{\text {rms }}$ distribution observed near the roughness element in Ref. 25 was an artifact of incorrect estimation of the wall location.

${ }^{35}$ O. Levin and D. S. Henningson, "Exponential vs algebraic growth and transition prediction in boundary layer flow," Flow, Turbul. Combust. 70, 183 (2003)

${ }^{36}$ C. E. Grosch and H. Salwen, "The continuous spectrum of the OrrSommerfeld equation. Part 1. The spectrum and the eigenfunctions," J. Fluid Mech. 87, 33 (1978).

${ }^{37}$ L. S. Hultgren and L. H. Gustavsson, "Algebraic growth of disturbances in a laminar boundary layer,” Phys. Fluids 24, 1000 (1981).

${ }^{38}$ M. Asai, M. Minagawa, and M. Nishioka, "The instability and breakdown of a near-wall low-speed streak," J. Fluid Mech. 455, 289 (2002).

${ }^{39}$ J. C. R. Hunt, C. J. Abell, J. A. Peterka, and H. Woo, "Kinematical studies of the flows around free or surface-mounted obstacles; applying topology to flow visualization," J. Fluid Mech. 86, 179 (1978).

${ }^{40}$ M. S. Acarlar and C. R. Smith, "A study of hairpin vortices in a laminar boundary layer. Part 1 . Hairpin vortices generated by a hemisphere protuberance," J. Fluid Mech. 175, 1 (1987).

${ }^{41}$ J. M. Dèlery, "Robert Legendre and Henry Werlé: Toward the elucidation of three-dimensional separation," Annu. Rev. Fluid Mech. 33, 129 (2001).

${ }^{42}$ A. Tumin and E. Reshotko, "The problem of boundary-layer flow encountering a three-dimensional hump revisited," AIAA Pap. 04-0101 (2004).

${ }^{43}$ E. B. White and F. G. Ergin, "Receptivity and transient growth of roughness-induced disturbances," AIAA Pap. 03-4243 (2003).

${ }^{44}$ M. Matsubara and P. H. Alfredsson, "Disturbance growth in boundary layers subjected to free stream turbulence," J. Fluid Mech. 430, 149 (2001).

${ }^{45}$ J. M. H. Fransson and P. H. Alfredsson, "On the disturbance growth in an asymptotic suction boundary layer," J. Fluid Mech. 482, 51 (2003). 